\title{
Middle segment pancreatectomy for a solid serous cystadenoma diagnosed by MRCP and review of the literature: A case report
}

\author{
YUICHIRO OKUMURA $^{1}$, TAKEHIRO NODA ${ }^{1}$, HIDETOSHI EGUCHI ${ }^{1}$, YOSHIFUMI IWAGAMI ${ }^{1}$, \\ DAISAKU YAMADA ${ }^{1}$, TADAFUMI ASAOKA ${ }^{1}$, KOICHI KAWAMOTO ${ }^{1}$, KUNIHITO GOTOH $^{1}$, \\ SHOGO KOBAYASHI ${ }^{1}$, KOJI UMESHITA ${ }^{2}$, YASUJI HASHIMOTO ${ }^{3}$, YUTAKA TAKEDA $^{4}$, \\ MASAHIRO TANEMURA ${ }^{5}$, MINORU SHIGEKAWA ${ }^{6}$, EIICHI MORII ${ }^{7}$, \\ TETSUO TAKEHARA $^{6}$, MASAKI MORI ${ }^{1}$ and YUICHIRO DOKI ${ }^{1}$ \\ ${ }^{1}$ Department of Gastroenterological Surgery and ${ }^{2}$ Division of Health Science, Graduate School of Medicine, \\ Osaka University, Osaka 565-0871; ${ }^{3}$ Department of Surgery, Yao Municipal Hospital, Osaka 581-0069; \\ ${ }^{4}$ Department of Surgery, Kansai Rosai Hospital, Hyogo 660-8511; ${ }^{5}$ Department of Surgery, Osaka Police \\ Hospital, Osaka 543-0035; Departments of ${ }^{6}$ Gastroenterology and Hepatology, and ${ }^{7}$ Department \\ of Pathology, Graduate School of Medicine, Osaka University, Osaka 565-0871, Japan
}

Received December 11, 2017; Accepted March 20, 2018

DOI: $10.3892 / \mathrm{mco} .2018 .1598$

\begin{abstract}
Solid serous cystadenoma of the pancreas is the rarest subtype of serous cystadenoma. Cystic structures are difficult to recognize by imaging studies. In the clinical setting, it is crucial to discriminate a solid serious cystadenoma from other solid pancreatic tumors. The present study reported a case of solid serous cystadenoma in which the magnetic resonance cholangiopancreatography (MRCP) findings were useful for diagnosis and decision-making regarding the surgical strategy, with a review of the previous reports of solid serous cystadenoma. A 50-year-old woman was referred to our hospital for investigation of a pancreatic body mass. A 2-cm hypervascular solid tumor was revealed by computed tomography. No typical radiological imaging findings of small cysts were detected, such as a honeycomb structure, and an adequate specimen could not be gained by biopsy under endoscopic ultrasonography. However, the tumor showed high
\end{abstract}

Correspondence to: Dr Hidetoshi Eguchi, Department of Gastroenterological Surgery, Graduate School of Medicine, Osaka University, 2-2 Yamadaoka E-2, Suita, Osaka 565-0871, Japan

E-mail: heguchi@gesurg.med.osaka-u.ac.jp

Abbreviations: CT, computed tomography; EUS, endoscopic ultrasonography; FNA, fine needle aspiration; MRCP, magnetic resonance cholangiopancreatography; MRI, magnetic resonance imaging; NET, neuroendocrine tumor; SCA, serous cystadenoma

Key words: serous cystadenoma, solid serous cystadenoma, serous cystic neoplasm, magnetic resonance cholangiopancreatography, pancreas intensity on MRCP, suggesting its cystic nature. A solid serous cystadenoma was suspected based on these radiological findings, and middle segment pancreatectomy was performed as a function-preserving surgery. The histological findings were compatible with a solid serous cystadenoma. In conclusion, MRCP imaging may be helpful for diagnosis and decisionmaking regarding the most appropriate surgical method for solid serous cystadenomas.

\section{Introductiom}

Serous cystadenoma (SCA) is fundamentally a multilobular cystic tumor that consists of a thin capsule and small cysts only millimeters in diameter. SCA constitutes approximately only 1 to $2 \%$ of all pancreatic tumors (1), but the number of patients with SCA has been growing due to the frequent use of radiography and recent improvements of imaging modalities. SCA is currently categorized into four subtypes: microcystic, macrocystic, mixed, and solid types. The most common subtype is microcystic type, and the cystic structure can be easily recognized in the former three subtypes. On the other hand, the solid type accounts for only $3 \%$ of all SCAs in a Japanese multicenter study (1), and it is described as noncystic, meaning that a cystic structure is too tiny to be detected macroscopically. Solid SCA is usually misdiagnosed preoperatively as neuroendocrine tumor (NET) (2). In several case reports, the authors reported that magnetic resonance cholangiopancreatography (MRCP) might be useful for preoperative diagnosis of SCA (3-5). However, it is generally considered difficult to preoperatively differentiate SCA from other solid pancreatic tumors by imaging modalities.

The present report describes a rare case of solid SCA in which the magnetic resonance imaging (MRI) findings were very informative in the diagnosis and decision to perform 
A

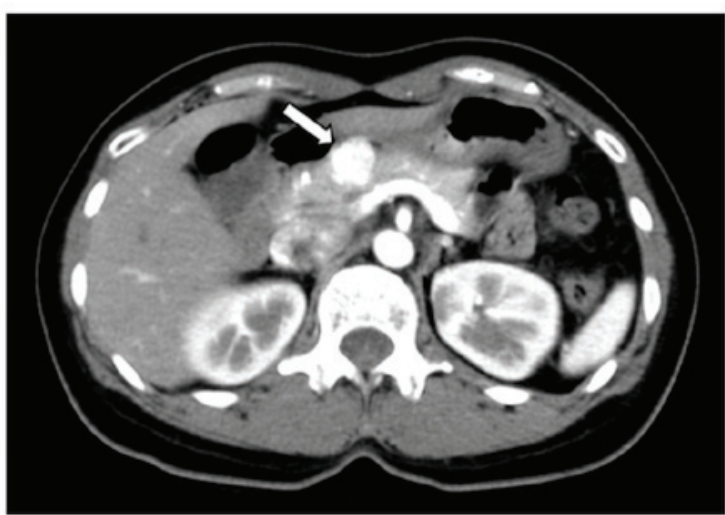

C

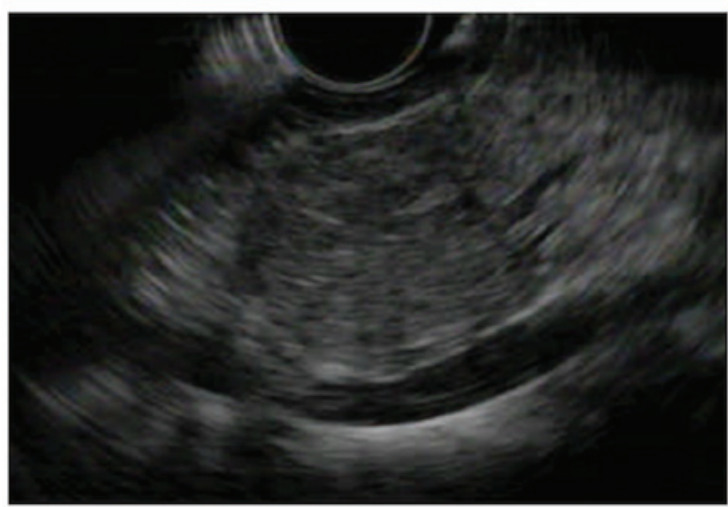

B

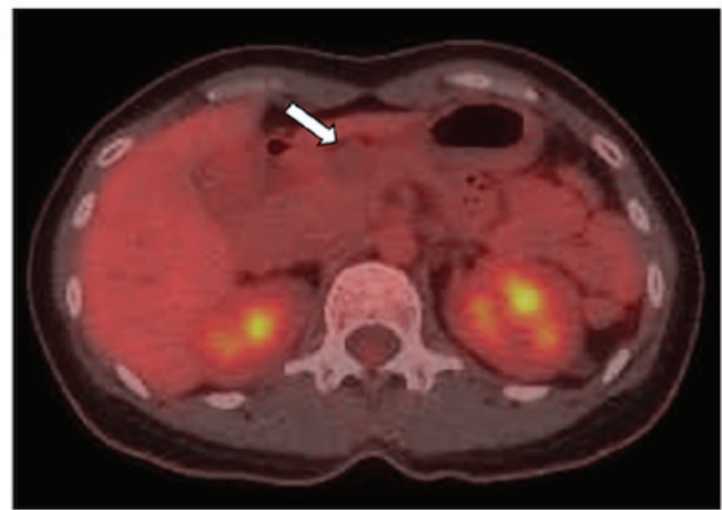

D

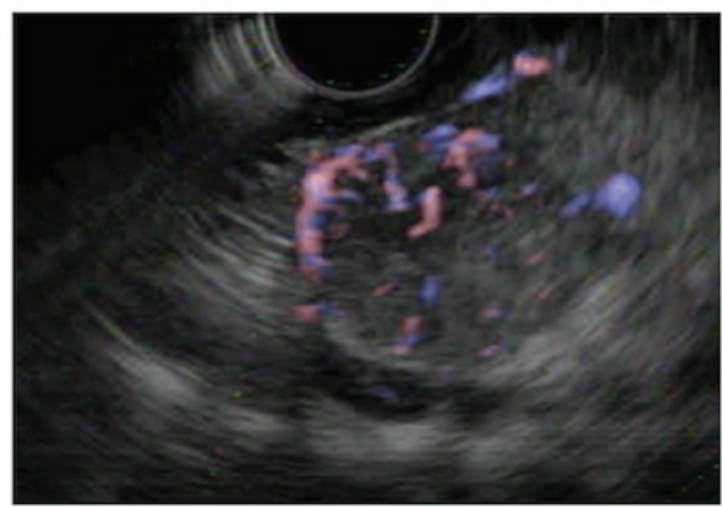

Figure 1. Computed tomography (CT) and abdominal ultrasonography. (A) Contrast-enhanced CT showed a well-enhanced solid tumor of 2 cm in the body of pancreas (arrow). (B) Positron emission tomography-CT showed no abnormal uptake (arrow). (C and D) Endoscopic ultrasonography showed a well circumscribed, hypoechoic, and hypervascular tumor without posterior echo enhancement.

middle segment pancreatectomy as an organ-preserving surgical strategy.

\section{Case report}

A 50-year-old woman was referred to the Department of Surgery in Osaka University Hospital for investigation of a pancreatic body mass detected during a health examination. She had no chief complaint. A benign thyroid tumor had been found, and she was followed up by annual ultrasonography. Physical examination revealed normal abdominal findings. Laboratory examination revealed no anemia, jaundice, or hyperglycemia. The serum level of carcinoembryonic antigen and carbohydrate antigen 19-9 were within the reference ranges, and no excess of pancreatic endocrine hormones, including insulin, glucagon, and gastrin, was observed.

Contrast-enhanced computed tomography (CT) demonstrated a $2-\mathrm{cm}$ solid mass in the body of the pancreas, which was strongly enhanced in the early phase (Fig. 1A). Fluorine-18 fluorodeoxyglucose positron emission tomography-CT showed no abnormal accumulation of the tracer in the tumor (Fig. 1B). Endoscopic ultrasonography (EUS) revealed a hypoechoic, heterogeneous, and hypervascular solid mass without posterior echo enhancement in the body of the pancreas, and no cystic component could be recognized (Fig. 1C and D). We performed EUS-guided fine needle aspiration (FNA) three times, but could not obtain adequate specimens for diagnosis because of contamination of blood. MRI clearly showed a round mass with low intensity on T1-weighted images (Fig. 2A) and high intensity on both
T2-weighted images (Fig.2B) and diffusion-weighted images (Fig. 2C). MRCP showed high intensity, and the tumor signal intensity was similar to that of an incidentally detected hepatic cyst (Fig. 2D). We strongly suspected the tumor to be a solid SCA based on the radiological findings, including MRCP, but histological confirmation could not be gained. We finally performed middle segment pancreatectomy as a functionpreserving surgery. The resected specimen was $2.6 \mathrm{~cm}$ in width, and surgical margin was ascertained by intraoperative ultrasonography (Fig. 3A). The cranial stump was cut off using a triple-row linear stapler and caudal stump was reconstructed by pancreaticogastrostomy using mattress sutures (6). The patient's recovery was complicated by pulmonary embolism (Grade 3 by Common Terminology Criteria for Adverse Events: CTCAE) and pancreatic fistula (Grade B by International Study Group of Pancreatic Fistula classification: ISGPF). The patient recovered with conservative treatment for pancreatic fistula and anti-coagulation therapy with warfarin for pulmonary embolism, and she was discharged on the 49 postoperative day.

The pancreatic tumor was clearly circumscribed within the resected specimen, and the cut surface of the tumor was solid, glossy, and reddish with a central fibrous scar in a stellate pattern (Fig. 3B). It was $2.2 \mathrm{~cm}$ in size, and no honeycomb structure characteristic of small cysts was detected macroscopically. Formalin-fixed paraffin-embedded tissue sections were prepared, and immunohistochemical staining was conducted using following antibodies; anti-mucin 6 antibody (Leica Biosystems, Nussloch, Germany), anti-synaptophysin antibody, anti-chromogranin antibody (both from Dako, Glostrup, 
A

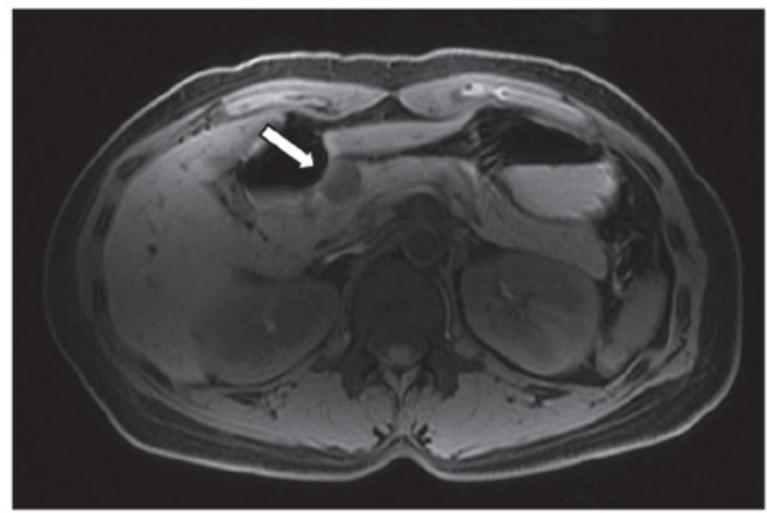

C

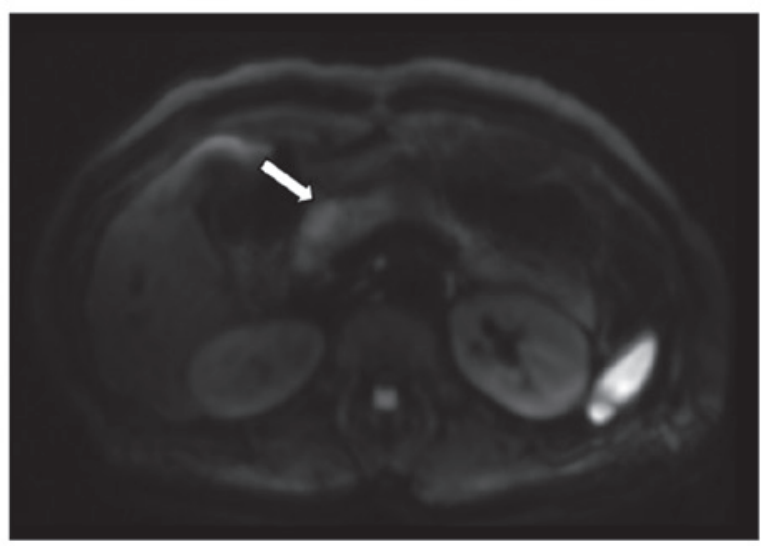

B

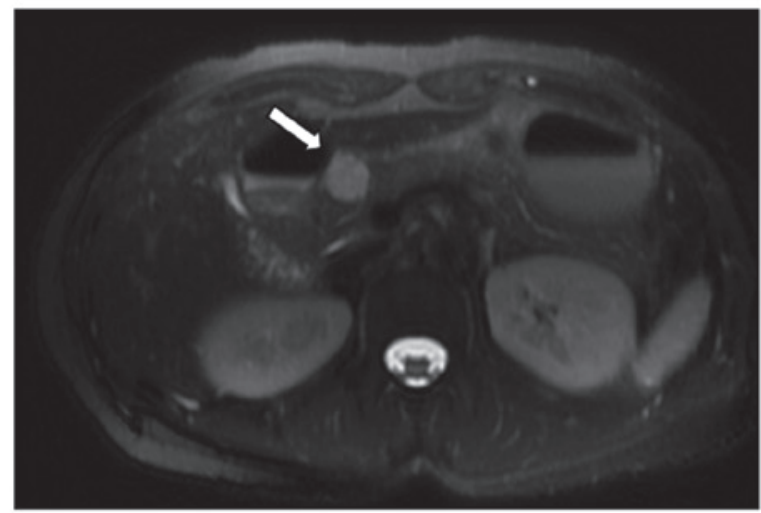

$\mathbf{D}$

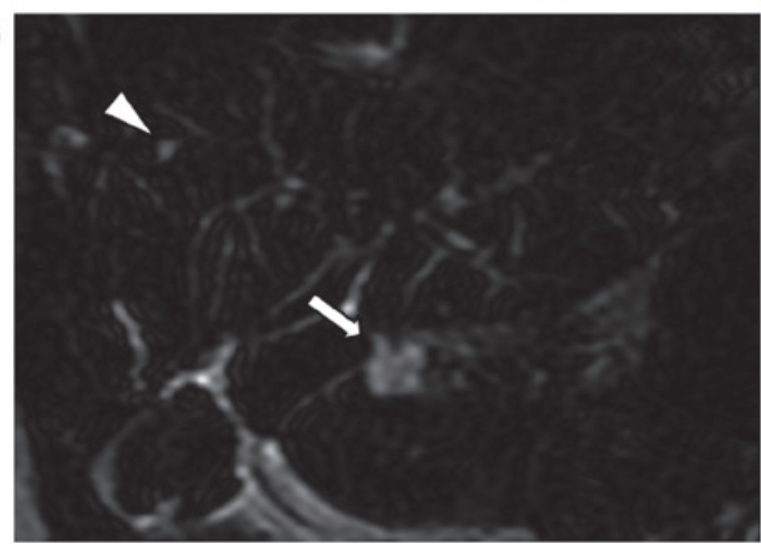

Figure 2. Magnetic resonance imaging. (A) low intensity on T1-weighted imaging (arrow), (B) high intensity on T2-weighted imaging (arrow), and (C) high intensity on diffusion-weighted imaging (arrow). (D) On a coronal magnetic resonance cholangiopancreatography image, the tumor showed high intensity (arrow), which was similar to that of the incidentally detected hepatic cyst (arrowhead).

A

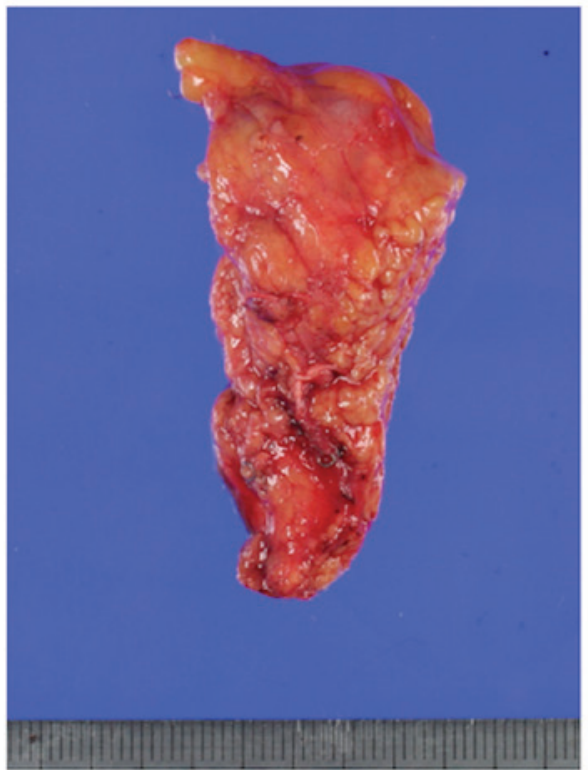

B

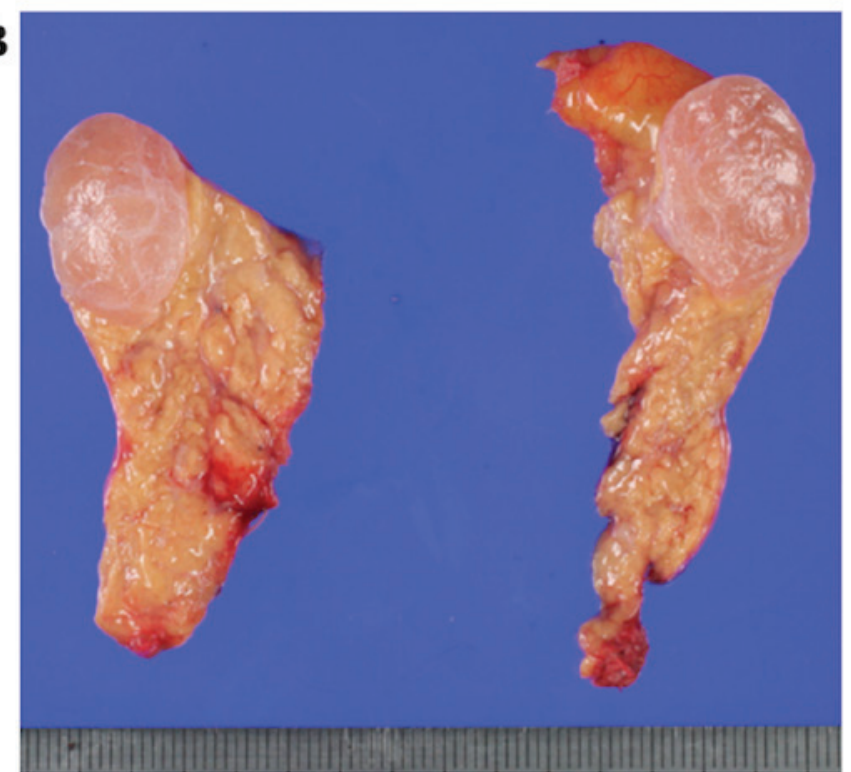

Figure 3. Resected specimen of the tumor. (A) Middle segment pancreatectomy was performed with enough surgical margin. (B) Macroscopic photograph of the pancreatic resection specimen, revealing a solid and glossy appearance with central fibrous tissue. The tumor measured $2.2 \mathrm{~cm}$.

Denmark) and anti-Ki-67 antibody (Roche Diagnostics, Basel, Switzerland). Briefly, the sections were incubated at room temperature with anti-mucin 6 for $32 \mathrm{~min}$, anti-synaptophysin for $16 \mathrm{~min}$, anti-chromogranin A for $16 \mathrm{~min}$ and anti-Ki-67 for $16 \mathrm{~min}$, respectively. Microscopic examination revealed numerous microcysts separated by hypocellular and dense collagen fibers. The inner surface of the cysts was lined by a single layer of cuboidal epithelium with clear cytoplasm (Fig. 4A). The cytoplasm was strongly stained by periodic acid-Schiff and digested by diastase because of the presence 
A

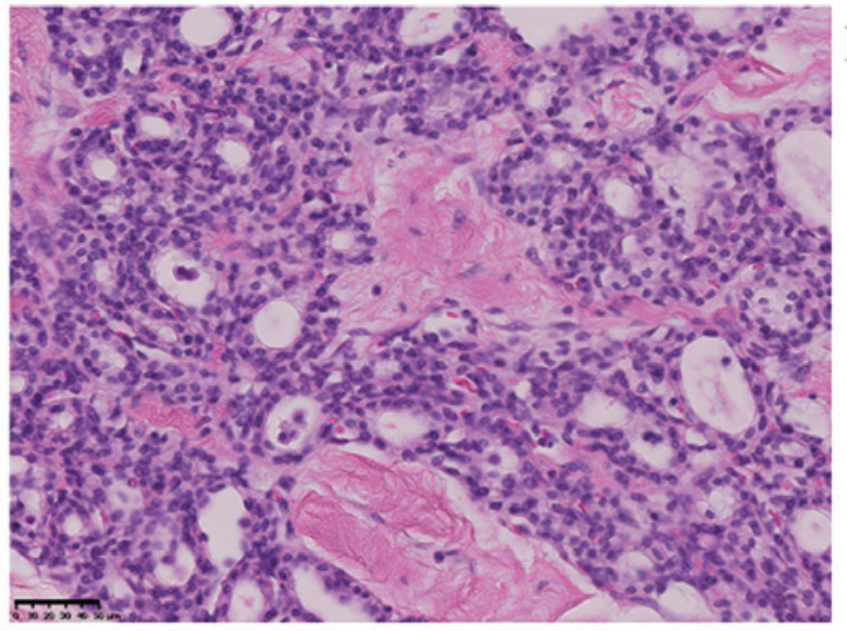

C

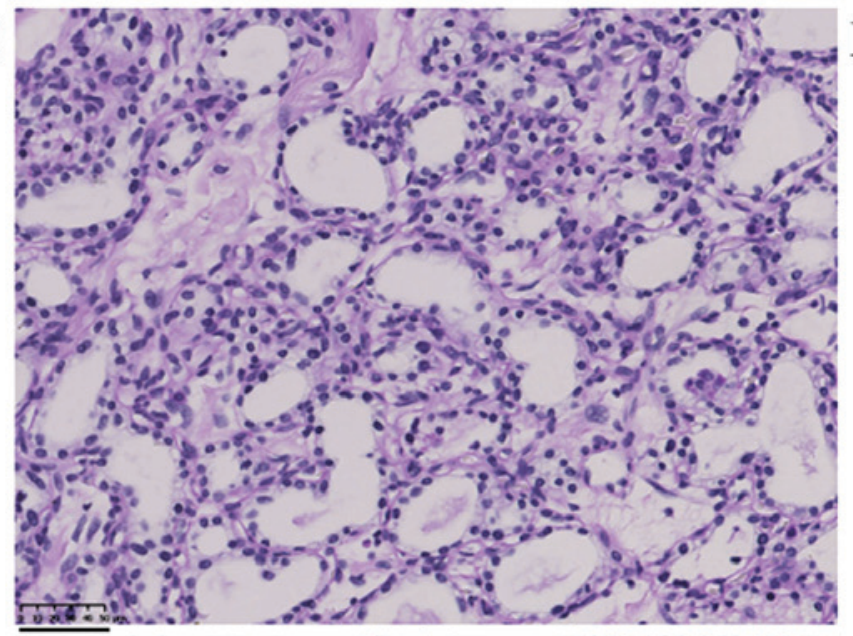

E

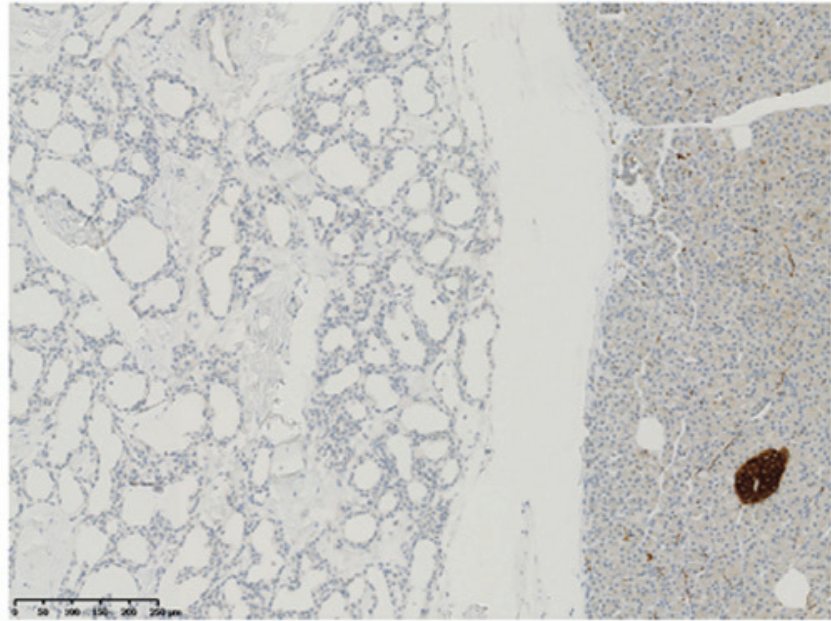

B

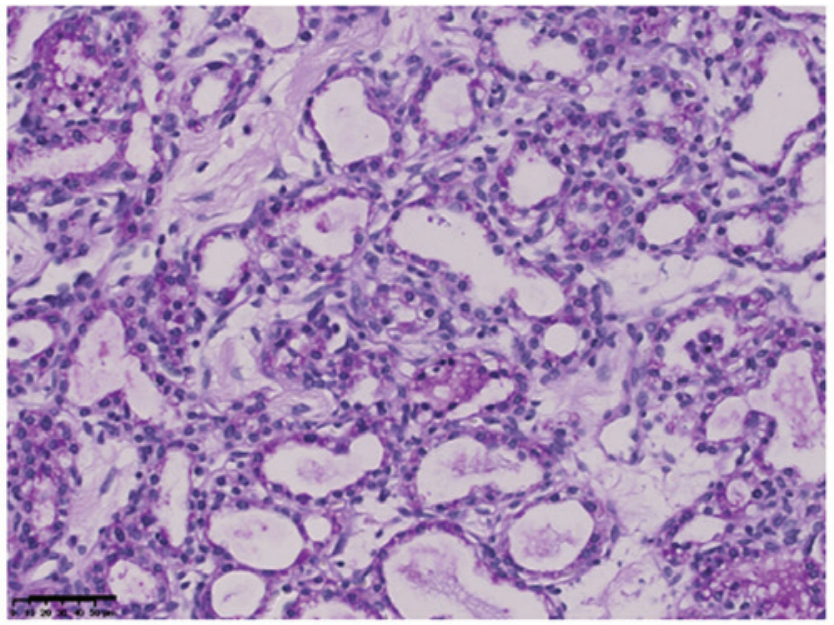

D

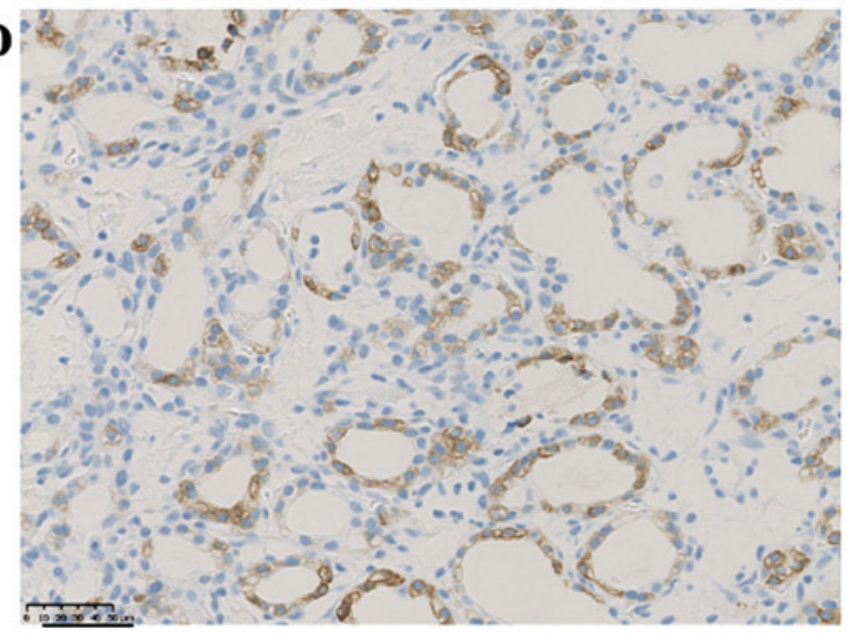

F

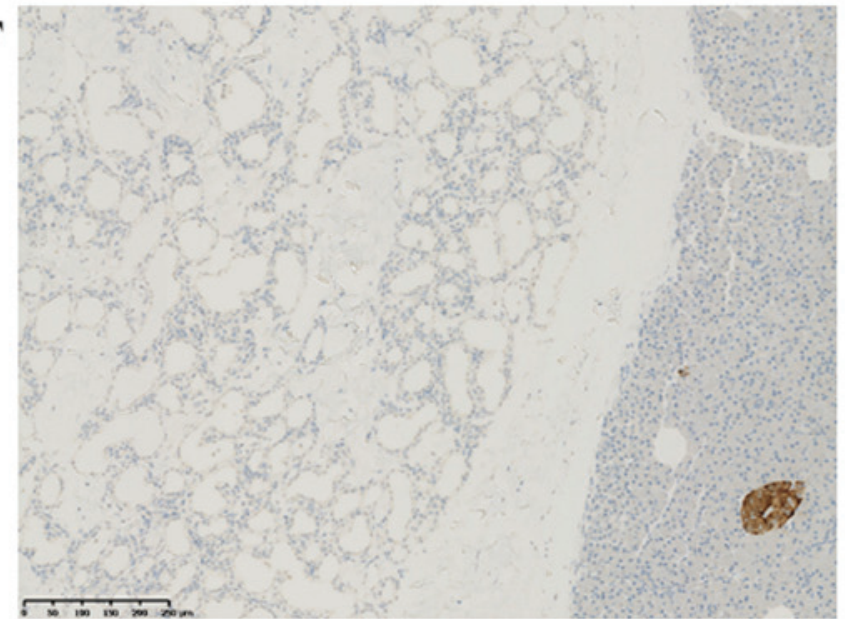

Figure 4. Histological examination of the resected specimen. (A) Microscopically, the tumor was composed of numerous tiny cysts lined by a single layer of cuboidal epithelium with abundant fibrous stroma (hematoxylin and eosin stain, $x 400)$. (B) The cytoplasm was strongly stained by periodic acid-Schiff stain (x400) and $(\mathrm{C})$ digested by diastase (x400). The cells were positive for (D) mucin 6 (x400) and negative for (E) synaptophysin (x200) and (F) chromogranin A (x200).

of abundant intracytoplasmic glycogen (Fig. 4B and C). The tumor cells were positive for mucin 6 (Fig. 4D) and negative for neuroendocrine differentiation labeling (synaptophysin (Fig. 4E) or chromogranin A (Fig. 4F). The Ki-67 labeling index was 1 to $2 \%$, and there was no evidence of malignancy or lymph node metastasis. The final diagnosis was a solid SCA. She had been in follow-up by every three months laboratory check and every six months radiological check by $\mathrm{CT}$. At the time of this writing (1 year postoperatively), the patient was clinically well with no evidence of recurrence. She had no diarrhea and weight loss without digestive enzymes. In addition, she also maintained favorable glucose tolerance without oral hypoglycemic agents or insulin. The preoperative and postoperative hemoglobin A1c level was not worsened (5.8 and 6.0\%, respectively). This clinical research was approved by the Research Ethics Board of the Osaka University Research Committee and conducted 


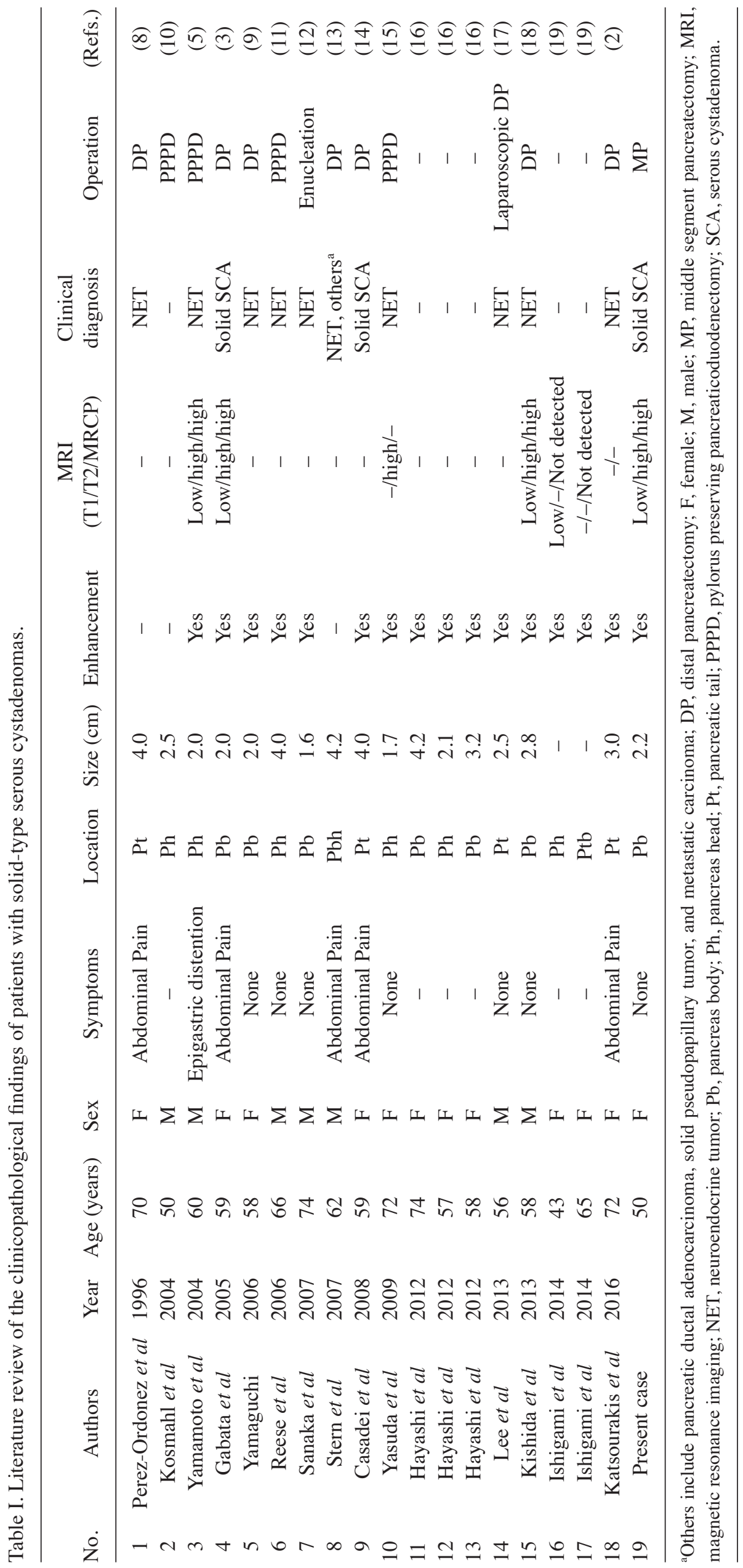


according to Institutional Review Board guidelines. Written informed consent was obtained from the patient prior to publication of the present case report.

\section{Discussion}

In 1978, Compagno and Oertel (7) first proposed the concept of serous cystic neoplasm of the pancreas. SCA is morphologically classified into four subtypes: microcystic, macrocystic, mixed, and solid types. The solid variant of SCA was first described by Perez-Ordonez et al (8) in 1996, who reported that the cells were arranged in small acini with no or minute central lumina, resembling a solid tumor. The characteristic radiological findings of SCA, such as a honeycomb appearance, polycystic pattern, lobularity, sunburst appearance (central calcification), and hemorrhage, are quite rare in solid SCA. Solid SCA is so rare that the incidence is only $3.0 \%$ of all SCAs, compared with microcystic type (58\%) and macrocystic type (20\%) (1). Whether solid SCA is a variant of SCA or a separate disease entity was historically controversial $(5,8,9)$, but it is now considered a variant of SCA because the cytological and immunohistological features of this tumor are very similar to those of SCA. Compared with the microcystic type, which is composed of numerous tiny cysts (usually $\sim 2-10 \mathrm{~mm}$ ), the solid type is formed by far smaller cysts that cannot be recognized by the naked eye and shows a homogeneous and glossy appearance.

We systematically reviewed the English literature by using PubMed and Google Scholar from 1995 to 2017. The keywords of 'solid serous cystadenoma', 'solid-type serous cystadenoma', 'serous cystic neoplasm' or 'solid serous adenoma' were used. We excluded the nonsurgical cases. To our knowledge, only 19 cases including our case have been published with a pathological confirmation (Table I) (2,3,5,8-19).

Some reports have described the radiological findings of solid SCA. A honeycomb structure is generally a typical finding of SCA; however, this finding cannot be detected in solid SCA even by EUS because the internal microlevel structure is difficult to capture. As a result, solid SCA is difficult to distinguish from other solid pancreatic tumors such as NET, acinar cell carcinoma, solid pseudopapillary tumor, and metastatic carcinoma. In most cases, NET is an especially important differential diagnosis because it is clinically the most common hypervascular solid tumor of the pancreas. Several case reports have shown that MRCP is a useful tool in distinguishing solid SCA from other solid pancreatic tumors (3-5). Solid SCA is reported to show T1 low intensity and $\mathrm{T} 2 \mathrm{high}$ intensity almost same as the other types of SCA (1). In general, MRCP involves heavily T2-weighted sequences, and the echo time is prolonged to about 10 times longer than regular T2-weighted imaging. On MRCP images, only a pure water cyst can gain hyperintensity. Both solid SCA and NET generally show hyperintensity on regular T2-weighted images. However, only solid SCA maintains this high intensity because of its cystic nature on MRCP. The high intensity on MRCP is quite a useful clue for clearly differentiating solid SCA from NET. MRI is becoming as common as CT for diagnostic modality. It can provide large amounts of valuable anatomical information preoperatively (20-22). If a tumor reveals high intensity on MRCP, this indicates that the tumor is composed mainly of not a solid substance but rather a watery fluid. In the present case, the signal intensity of the tumor was similar to that of the incidentally detected hepatic cyst. This finding could also be valuable for reaching the accurate preoperative diagnosis.

With respect to treatment, expectant management has been proposed when SCA is small and definitely diagnosed because this neoplasm grows slowly and there is a minimal risk of malignant transformation (23). When nonoperative management is selected, histological examination by EUS-FNA is recommended to provide essential information in advance, but an adequate specimen can be rarely obtained by EUS-FNA.

The role of EUS-FNA is to obtain preoperative cytological confirmation of pancreatic malignant tumors (24), and tumor markers and DNA analysis of cyst aspirates are reportedly helpful in improving diagnostic yield (25). EUS-FNA is recommended for NET as an informative tool in differentiating from other pancreatic tumors because of its high diagnostic sensitivity, which is reported as high as $87-90 \%$ (26-28). However, the reported diagnostic accuracy of EUS-guided biopsy for cystic tumors of the pancreas ranges from 17-21\% (18,29-31), and the specimen often lacks the epithelial tissue necessary for diagnosis because of its cystic nature. Consequently, EUS-FNA alone is unlikely to provide the high level of diagnostic evidence necessary to support observational management. The indication for EUS about solid SCA is not mentioned because of its rarity in the guidelines about pancreatic cysts $(25,32-34)$. In the present case, the tumor was initially deemed a NET because of its hypervascularity and solid appearance. However, the MRCP finding strongly indicated the possibility of solid SCA. We tried to gain histological confirmation by EUS-FNA, but ended up as a failure, and the possibility of NET could not be excluded. We finally selected surgical intervention because solid SCA is rare and the final diagnosis ought to be based on histological confirmation of a surgical specimen unless the diagnosis is preoperatively assured by EUS-FNA.

We performed middle segment pancreatectomy as a function-preserving surgery because the tumor was as small as $2.2 \mathrm{~cm}$. An organ-preserving surgical procedure is generally recommended for SCA because lymph node metastasis of SCA is quite rare and lymphadenectomy is not necessary (35). In previous reports of SCA with clear mention about operative procedure, all of the 13 patients underwent pancreaticoduodenectomy, distal pancreatectomy, or enucleation (Table I). Previous five cases lacked in operation method, as those reports placed value in the imaging pitfalls of solid SCA. Therefore, Our case is the first in which middle segment pancreatectomy was performed for a solid SCA as an organ-preserving surgery, and this procedure might lead to better preservation of endocrine function (36).

Solid SCA of the pancreas is definitively a rare disease, but oncologic surgeons should be aware of the characteristics of this neoplasm to allow for a correct preoperative diagnosis. Further investigation to accumulate more evidence regarding this rare disease is expected.

We experienced a case of solid SCA of the pancreas for which MRCP imaging was very helpful for accurate diagnosis and decision-making regarding the surgical method.

\section{Acknowledgements}

Not applicable. 


\section{Funding}

No funding was received.

\section{Availability of data and materials}

The datasets used during the current study are available from the corresponding author on reasonable request.

\section{Authors' contributions}

YO, TN, HE, MS, TT and YD conceived and designed this study. YO, YI, DY, TA, KK, KG and EM acquired the data. YO, SK, KU, YH, YT, MT and MM drafted the manuscript. All authors read and approved the final manuscript.

\section{Ethics approval and consent to participate}

This clinical research was approved by the Research Ethics Board of the Osaka University Research Committee and conducted according to Institutional Review Board guidelines. Written informed consent was obtained from the patient prior to publication of the present case report.

\section{Consent for publication}

Written informed consent was obtained from the patient involved in this publication and accompanying images. A copy of this written consent is available for review by the Editor-inChief of this journal.

\section{Competing interests}

The authors declare that they have no competing interests.

\section{References}

1. Kimura W, Moriya T, Hirai I, Hanada K, Abe H, Yanagisawa A, Fukushima N, Ohike N, Shimizu M, Hatori T, et al: Multicenter study of serous cystic neoplasm of the Japan pancreas society. Pancreas 41: 380-387, 2012.

2. Katsourakis A, Dimitriou I, Noussios G, Chatzis I and Chatzitheoclitos E: Solid Serous Adenoma of the Pancreas: A Case Report and Review of the Literature. Case Rep Surg 2016: 3730249, 2016.

3. Gabata T, Terayama N, Yamashiro M, Takamatsu S, Yoshida K, Matsui O, Usukura M, Takeshita M and Minato H: Solid serous cystadenoma of the pancreas: MR imaging with pathologic correlation. Abdom Imaging 30: 605-609, 2005.

4. Machado MC and Machado MA: Solid serous adenoma of the pancreas: an uncommon but important entity. Europ J Surg Oncol 34: 730-733, 2008

5. Yamamoto T, Takahashi N, Yamaguchi T and Imamura Y: A case of solid variant type of pancreatic serous cystadenoma mimicking islet cell tumor. Clin Imaging 28: 49-51, 2004.

6. Ohigashi H, Ishikawa O, Eguchi H, Sasaki Y, Yamada T, Kishi K Noura S, Takachi K, Miyashiro I, Oue M, et al: A simple and safe anastomosis in pancreaticogastrostomy using mattress sutures. Am J Surg 196: 130-134, 2008.

7. Compagno J and Oertel JE: Microcystic adenomas of the pancreas (glycogen-rich cystadenomas): A clinicopathologic study of 34 cases. Am J Clin Pathol 69: 289-298, 1978.

8. Perez-Ordonez B, Naseem A, Lieberman PH and Klimstra DS : Solid serous adenoma of the pancreas. The solid variant of serous cystadenoma? Am J Surg Pathol 20: 1401-1405, 1996.

9. Yamaguchi M: Solid serous adenoma of the pancreas: A solid variant of serous cystadenoma or a separate disease entity? J Gastroenterol 41: 178-179, 2006.
10. Kosmahl M, Wagner J, Peters K, Sipos B and Klöppel G: Serous cystic neoplasms of the pancreas: An immunohistochemical analysis revealing alpha-inhibin, neuron-specific enolase, and MUC6 as new markers. Am J Surg Pathol 28: 339-346, 2004.

11. Reese SA, Traverso LW, Jacobs TW and Longnecker DS: Solid serous adenoma of the pancreas: A rare variant within the family of pancreatic serous cystic neoplasms. Pancreas 33: 96-99, 2006.

12. Sanaka MR, Kowalski TE, Brotz C, Yeo CJ, McCue P and Palazzo J: Solid serous adenoma of the pancreas: A rare form of serous cystadenoma. Dig Dis Sci 52: 3154-3156, 2007.

13. Stern JR, Frankel WL, Ellison EC and Bloomston M: Solid serous microcystic adenoma of the pancreas. World J Surg Oncol 5: 26, 2007.

14. Casadei R, D'Ambra M, Pezzilli R, Ricci C, Calculli L, Lega S, Antonacci N, Monari F and Minni F: Solid serous microcystic tumor of the pancreas. JOP 9: 538-540, 2008

15. Yasuda A, Sawai H, Ochi N, Matsuo Y, Okada Y and Takeyama H: Solid variant of serous cystadenoma of the pancreas. Arch Med Sci 7: 353-355, 2011

16. Hayashi K, Fujimitsu R, Ida M, Sakamoto K, Higashihara H, Hamada Y and Yoshimitsu K: CT differentiation of solid serous cystadenoma vs endocrine tumor of the pancreas. Eur J Radiol 81: e203-e208, 2012.

17. Lee SD, Han SS and Hong EK: Solid serous cystic neoplasm of the pancreas with invasive growth. J Hepatobiliary Pancreat Sci 20: 454-456, 2013.

18. Kishida Y, Matsubayashi H, Okamura Y, Uesaka K, Sasaki K, Sawai H, Imai K and Ono H: A case of solid-type serous cystadenoma mimicking neuroendocrine tumor of the pancreas. J Dig Dis 15: 211-215, 2014.

19. Ishigami K, Nishie A, Asayama Y, Ushijima Y, Takayama Y, Fujita N, Takahata S, Ohtsuka T, Ito T, Igarashi H, et al: Imaging pitfalls of pancreatic serous cystic neoplasm and its potential mimickers. World J Radiol 6: 36-47, 2014

20. Shimizu Y, Otani T, Matsumoto J, Takanishi K, Minami T, Tsunoda $\mathrm{H}$ and Miyazaki M: Cystic duct with no visible signal on magnetic resonance cholangiography is associated with laparoscopic difficulties: An analysis of 695 cases. Surg Today 44: 1490-1495, 2014

21. Sueda T, Ohue M, Noura S, Shingai T, Nakanishi K and Yano M: Prognostic significance of a preoperative magnetic resonance imaging assessment of the distance of mesorectal extension in clinical T3 lower rectal cancer. Surg Today 46: 1249-1257, 2016.

22. Kolodziejczyk E, Jurkiewicz E, Pertkiewicz J, Wejnarska K, Dadalski M, Kierkus J, Woynarowski M, Ryzko J and Oracz G: MRCP Versus ERCP in the Evaluation of Chronic Pancreatitis in Children: Which Is the Better Choice? Pancreas 45: 1115-1119, 2016.

23. Tseng JF, Warshaw AL, Sahani DV, Lauwers GY, Rattner DW and Castillo CF-d: Serous Cystadenoma of the Pancreas. Transactions of the ... Meeting of the American Surgical Association 123: 111-118, 2005

24. Ohtsuka T, Tamura K, Ideno N, Aso T, Nagayoshi Y, Kono H, Ueda J, Takahata S, Aso A, Igarashi H, et al: Role of ERCP in the era of EUS-FNA for preoperative cytological confirmation of resectable pancreatic ductal adenocarcinoma. Surg Today 44: 1887-1892, 2014.

25. Chiang AL and Lee LS: Clinical approach to incidental pancreatic cysts. World J Gastroenterol 22: 1236-1245, 2016.

26. Figueiredo FA, Giovannini M, Monges G, Bories E, Pesenti C, Caillol F and Delpero JR: EUS-FNA predicts 5-year survival in pancreatic endocrine tumors. Gastrointest Endosc 70: 907-914, 2009.

27. Pais SA, Al-Haddad M, Mohamadnejad M, Leblanc JK, Sherman S, McHenry L and DeWitt JM: EUS for pancreatic neuroendocrine tumors: A single-center, 11-year experience. Gastrointest Endosc 71: 1185-1193, 2010.

28. Gornals J, Varas M, Catala I, Maisterra S, Pons C, Bargallo D, Serrano T and Fabregat J: Definitive diagnosis of neuroendocrine tumors using fine-needle aspiration-puncture guided by endoscopic ultrasonography. Rev Esp Enferm Dig 103: 123-128, 2011.

29. Brugge WR: Role of endoscopic ultrasound in the diagnosis of cystic lesions of the pancreas. Pancreatology 1: 637-640, 2001.

30. Belsley NA, Pitman MB, Lauwers GY, Brugge WR and Deshpande V: Serous cystadenoma of the pancreas: Limitations and pitfalls of endoscopic ultrasound-guided fine-needle aspiration biopsy. Cancer 114: 102-110, 2008.

31. Huang P, Staerkel G, Sneige N and Gong Y: Fine-needle aspiration of pancreatic serous cystadenoma: Cytologic features and diagnostic pitfalls. Cancer 108: 239-249, 2006. 
32. Vege SS, Ziring B, Jain R, Moayyedi P, Clinical Guidelines C and American Gastroenterology A: American gastroenterological association institute guideline on the diagnosis and management of asymptomatic neoplastic pancreatic cysts. Gastroenterology 148: 819-822; quize812-813, 2015.

33. Tanaka M, Chari S, Adsay V, Fernandez-del Castillo C, Falconi M, Shimizu M, Yamaguchi K, Yamao K and Matsuno S; International Association of Pancreatology: International consensus guidelines for management of intraductal papillary mucinous neoplasms and mucinous cystic neoplasms of the pancreas. Pancreatology 6: 17-32, 2006.

34. Tanaka M, Chari S, Adsay V, Fernandez-del Castillo C, Falconi M, Shimizu M, Yamaguchi K, Yamao K and Matsuno S; International Association of Pancreatology: International consensus guidelines for management of intraductal papillary mucinous neoplasms and mucinous cystic neoplasms of the pancreas. Pancreatology 6: 17-32, 2006.
35. Galanis C, Zamani A, Cameron JL, Campbell KA, Lillemoe KD, Caparrelli D, Chang D, Hruban RH and Yeo CJ: Resected serous cystic neoplasms of the pancreas: A review of 158 patients with recommendations for treatment. J Gastrointest Surg 11: 820-826, 2007.

36. Colvin H, Mizushima T, Eguchi H, Takiguchi S, Doki Y and Mori M: Gastroenterological surgery in Japan: The past, the present and the future. Ann Gastroenterol Surg 1: 5-10, 2017.

This work is licensed under a Creative Commons Attribution-NonCommercial-NoDerivatives 4.0 International (CC BY-NC-ND 4.0) License. 